\title{
CMS Muon System Phase 2 Upgrade with triple-GEM detectors
}

D. Abbaneo ${ }^{18}$, M. Abbas ${ }^{18}$, M. Abbrescia ${ }^{2}$, M. Abi Akl ${ }^{14}$, O. Aboamer ${ }^{8}$, D. Acosta ${ }^{16}$, A. Ahmad ${ }^{20}$, W. Ahmed ${ }^{20}$, A. Aleksandrov2 ${ }^{29}$ P. Altieri2 ${ }^{2}$, C. Asawatangtrakuldee ${ }^{3}$, P. Aspell ${ }^{18}$, Y. Assran ${ }^{8}$, I. Awan ${ }^{20}$, S. Bally ${ }^{18}$, Y. Ban $^{3}$, S. Banerjee $^{21}$, V. Barashko ${ }^{16}$, P. Barria ${ }^{5}$, G. Bencze ${ }^{7}$, N. Beni ${ }^{11}$, L. Benussi ${ }^{15}$, V. Bhopatkar ${ }^{24}$, S. Bianco ${ }^{15}$, J. Bos ${ }^{18}$, O. Bouhali $^{14}$, A. Braghieri ${ }^{27}$, S. Braibant ${ }^{4}$, S. Buontempo ${ }^{26}$, C. Calabria ${ }^{2}$, M. Caponero ${ }^{15}$, C. Caputo ${ }^{2}$, F. Cassese ${ }^{26}$, A. Castaneda $^{26}$, S. Cauwenbergh ${ }^{19}$, F.R. Cavallo ${ }^{4}$, A. Celik ${ }^{10}$, M. Choi ${ }^{33}$, S. Choi ${ }^{31}$, J. Christiansen ${ }^{18}$, A. Cimmino ${ }^{19}$, S.

Colafranceschi1 $^{18}$, A. Colaleo ${ }^{2}$, A. Conde Garcia ${ }^{18}$, S. Czellar ${ }^{11}$, M.M. Dabrowski ${ }^{18}$, G. De Lentdecker ${ }^{5}$, R. De

Oliveira $^{18}$, G. de Robertis ${ }^{2}$, S. Dildick ${ }^{10,19}$, B. Dorney ${ }^{18}$, G. Endroczi ${ }^{7}$, F. Errico ${ }^{2}$, A. Fenyvesi ${ }^{11}$, S. Ferry ${ }^{18}$, I. Furic ${ }^{16}$,

P. Giacomelli4 ${ }^{4}$, J. Gilmore ${ }^{10}$, V. Golovtsov ${ }^{17}$, M.Gruchala ${ }^{35}$, L. Guiducci ${ }^{18}$, F. Guilloux ${ }^{28}$, A. Gutierrez ${ }^{13}$, R.M.

Hadjiiska $^{29}$, J. Haus ${ }^{23}$, K. Hoepfner ${ }^{1}$, M. Hohlmann ${ }^{24}$, H. Hoorani ${ }^{20}$, P. Iaydjiev ${ }^{29}$, Y.G. Jeng ${ }^{33}$, T. Kamon ${ }^{10}$, P.

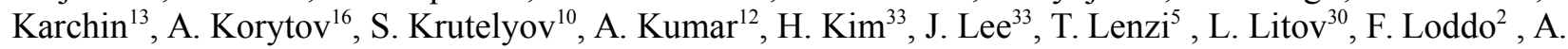

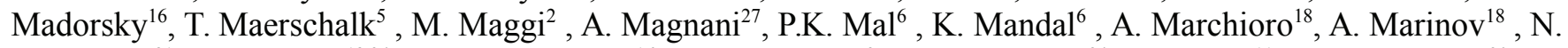
Majumdar $^{21}$, J.A. Merlin ${ }^{18,34}$, G. Mitselmakher ${ }^{16}$, A.K. Mohanty ${ }^{25}$, A. Mohapatra ${ }^{24}$, J. Molnar ${ }^{11}$, S. Muhammad ${ }^{20}$, S. Mukhopadhyay $^{21}$, M. Naimuddin ${ }^{12}$, S. Nuzzo ${ }^{2}$, E. Oliveri ${ }^{18}$, L.M. Pant ${ }^{25}$, P. Paolucci ${ }^{26}$, I. Park ${ }^{33}$, G. Passeggio ${ }^{26}$, B.

Pavlov $^{30}$, B. Philipps ${ }^{1}$, D. Piccolo ${ }^{15}$, H. Postema ${ }^{18}$, A. Puig Baranac ${ }^{18}$, A. Radi ${ }^{8}$, R. Radogna ${ }^{2}$, G. Raffone ${ }^{15}$, A.

Ranieri $^{2}$, G. Rashevski ${ }^{29}$, C. Riccardi ${ }^{27}$, M. Rodozov ${ }^{29}$, A. Rodrigues $1{ }^{18}$ L. Ropelewski ${ }^{18}$, S. RoyChowdhury ${ }^{21}$, G.

Ryu $^{33}$, M.S. Ryu ${ }^{33}$, A. Safonov ${ }^{10}$, S. Salva ${ }^{19^{*}}$, G. Saviano ${ }^{15}$, A. Sharma ${ }^{2}$ A. Sharma ${ }^{18}$, R. Sharma ${ }^{12}$, A.H. Shah ${ }^{12}$, M. Shopova $^{29}$, J. Sturdy ${ }^{13}$, G. Sultanov ${ }^{29}$, S.K. Swain ${ }^{6}$, Z. Szillasi ${ }^{11}$, J. Talvitie2 ${ }^{22}$ A. Tatarinov ${ }^{10}$, T. Tuuva ${ }^{22}$, M. Tytgat ${ }^{19}$,

I. Vai ${ }^{27}$, M. Van Stenis ${ }^{18}$, R. Venditti ${ }^{2}$, E. Verhagen ${ }^{5}$, P. Verwilligen ${ }^{2}$, P. Vitulo ${ }^{27}$, S. Volkov $^{17}$, A. Vorobyev ${ }^{17}$, D. Wang $^{3}$, M. Wang ${ }^{3}$, U. Yang ${ }^{32}$, Y. Yang ${ }^{5}$, R. Yonamine ${ }^{5}$, N. Zaganidis ${ }^{19}$, F. Zenoni ${ }^{5}$, and A. Zhang ${ }^{24}$

Manuscript received December 5, 2015.

1 RWTH Aachen University, III Physikalisches Institut A, Aachen, Germany

2 INFN Bari and University of Bari, Bari, Italy

3 Peking University, Beijing, China

4 INFN Bologna and University of Bologna, Bologna, Italy

5 Universite Libre de Bruxelles, Brussels, Belgium

6 National Institute of Science Education and Research, Bhubaneswar

7 Institute for Particle and Nuclear Physics, Wigner Research Centre for

Physics, Hungarian Academy of Sciences, Budapest, Hungary

8 Academy of Scientific Research and Technology - Egyptian Network of

High Energy Physics, ASRT-ENHEP, Cairo, Egypt

9 Helwan University \& CTP, Cairo, Egypt

10 Texas A\&M University, College Station, U.S.A.

11 Institute for Nuclear Research of the Hungarian Academy of Sciences

(ATOMKI), Debrecen, Hungary

12 University of Delhi, Delhi, India

13 Wayne State University, Detroit, U.S.A

14 Texas A\&M University at Qatar, Doha, Qatar

15 Laboratori Nazionali di Frascati - INFN, Frascati, Italy

16 University of Florida, Gainesville, U.S.A.

17 Petersburg Nuclear Physics Institute, Gatchina, Russia

18 CERN, Geneva, Switzerland

19 Ghent University, Dept. of Physics and Astronomy, Ghent, Belgium

20 National Center for Physics, Quaid-i-Azam University Campus, Islamabad,

Pakistan

21 Saha Institute of Nuclear Physics, Kolkata, India

22 Lappeenranta University of Technology, Lappeenranta, Finland

23 University of California, Los Angeles, U.S.A.

24 Florida Institute of Technology, Melbourne, U.S.A.

25 Bhabha Atomic Research Centre, Mumbai, India

26 INFN Napoli, Napoli, Italy

27 INFN Pavia and University of Pavia, Pavia, Italy

28 IRFU CEA-Saclay, Saclay, France

29 Institute for Nuclear Research and Nuclear Energy, Sofia, Bulgaria

30 Sofia University, Sofia, Bulgaria

31 Korea University, Seoul, Korea

32 Seoul National University, Seoul, Korea

33 University of Seoul, Seoul, Korea
34 Institut Pluridisciplinaire - Hubert Curien (IPHC), Strasbourg, France

35 Tadeusz Kosciuszko University of Technology, Poland

* Corresponding author: sinem.salva@ugent.be

\begin{abstract}
The Compact Muon Solenoid (CMS) detector installed at the CERN Large Hadron Collider (LHC) has an extensive muon system which provides information simultaneously for identification, track reconstruction and triggering of muons. As a consequence of the extreme particle rate and high integrated charge, the essentiality to upgrade the LHC has given rise to the High Luminosity phase of the LHC (HL-LHC) project so that the CMS muon system will be upgraded with superior technological challenges. The CMS GEM collaboration offers a solution to equip the high-eta region of the muon system for Phase 2 (after the year 2017) with large-area triple-layer Gas Electron Multiplier (GEM) detectors, since GEMs have the ability to provide robust and redundant tracking and triggering functions with an excellent spatial resolution of order 100 micron and a high particle rate capability, with a close to $100 \%$ detection efficiency. In this contribution, the present status of the triple-GEM project will be reviewed, and the significant achievements from the start of the R\&D in 2009 will be emphasized.
\end{abstract}

\section{INTRODUCTION}

$\mathrm{T}$ The CMS Muon System, which is designed to detect and reconstruct muons with the best precision, needs an upgrade to handle the high rate and intensive environment of High Luminosity LHC during Phase 2. New detector requirements include a high rate capability $\left(\sim \mathrm{Mhz} / \mathrm{cm}^{2}\right)$, and in addition, a new station must be able to 
survive the high radiation background, where the charge expected to be integrated in 20 years of operation of the LHC is around $100 \mathrm{mC} / \mathrm{cm}^{2}$. The current detector design is not sustainable at the higher rate and background, therefore new technologies have been considered, in particular Gas Electron Multiplier (GEM) [1] detectors. The installation of GEMs (labeled as GE1/1 in Fig. 1), which are approved for the second LHC long shutdown, will equip the inner endcap station, while the installation of the more recent GEMs (labeled as GE2/1 and ME0 in Fig. 1), which are proposed for the third LHC long shutdown [2], would equip the second endcap stations. This contribution presents only the results of a research on the GE1/1. The installation of GE1/1 station in the forward region $1.6<|\eta|<2.2$, where $\eta$ is taken as the pseudorapidity defined as $\eta=-\ln (\tan (\theta / 2))$; with $\theta$ being the polar angle, is expected to fulfill the mentioned requirements.

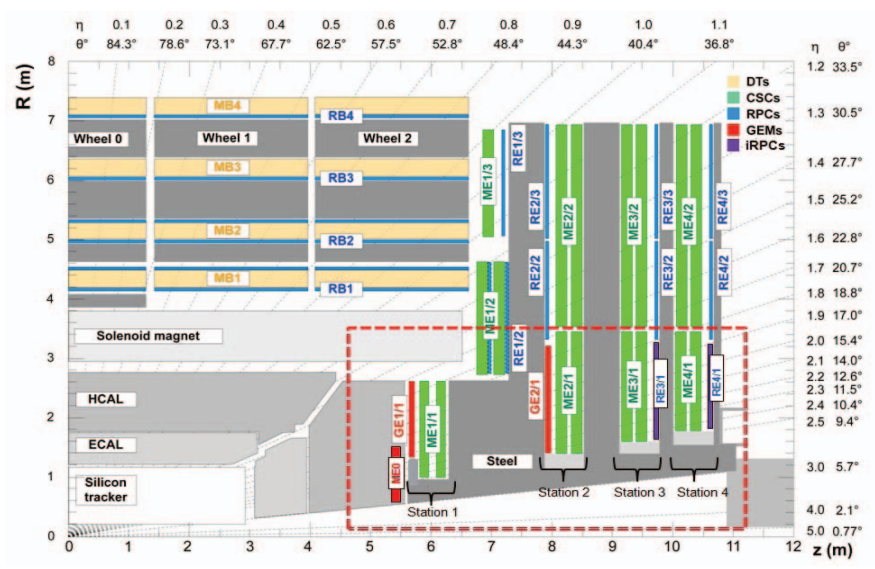

Fig. 1. A quadrant of the CMS muon system with different subsystems. The stations of the forward detectors are shown in the red box with GE1/1, GE2/1 and ME0.

\section{BENEFITS OF GE1/1 TO THE MUON SYSTEM}

GE1/1 allows measurement of muon bending angle at trigger level, which original muon system cannot measure. The muon lever-arm between the GEMs and the adjacent Cathode Strip Chambers (CSCs) will allow to determine the muon $\mathrm{p}_{\mathrm{T}}$ by measuring the bending angle due to the magnetic field in the first muon station. This measurement helps in reducing the rate of soft muons that pass the trigger threshold. According to simulations using the official CMS framework, it allows to obtain acceptable trigger rates without increasing the $\mathrm{p}_{\mathrm{T}}$ threshold. The importance of keeping low p thresholds has been investigated in various benchmark physics channels. In the High Luminosity LHC era, the CSCs installed in the forward region will have been operating for more than ten years in an extreme radiation environment. Dedicated simulation studies indicate that GE1/1 would guarantee a stable trigger efficiency even with a fraction of damaged
CSCs. Also the muon reconstruction performance is expected to improve with the implementation of GE1/1, especially in case of ageing problems in the CSCs. Fig. 2 shows the consequent reduction in the trigger rate [3].

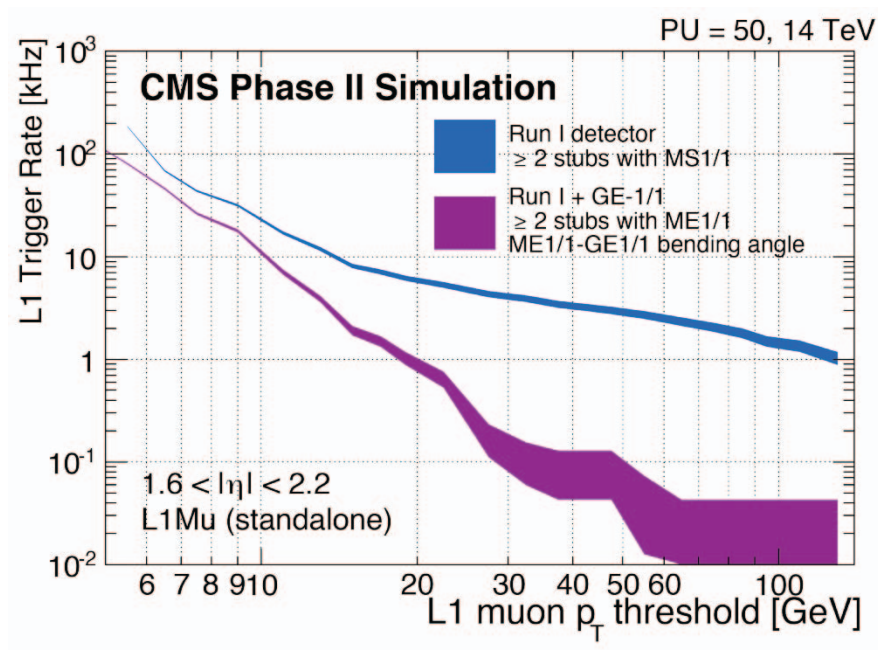

Fig. 2. Level-1 muon trigger rates before and after the GE1/1 upgrade at a luminosity of $2 \times 10^{34} \mathrm{~cm}^{-2} \mathrm{~s}^{-1}$, for constant efficiency of $94 \%$.

\section{GEM ENDCAP SYSTEM}

The first CMS muon endcap station where the inner ring will be equipped with 18 long and 18 short triple-GEM superchambers (a pair of GEM chambers form one superchamber). The prototypes for GEM Endcap Station 1 Ring 1 system are large-area trapezoidal shaped detectors,

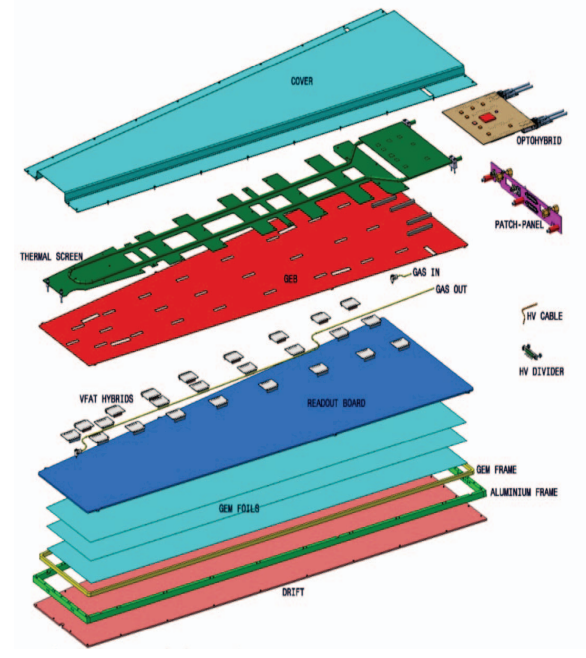

(a)

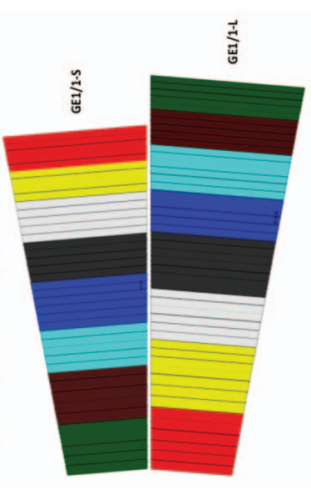

(b)
Fig. 3. (a) The mechanical design of a single trapezoidal GE1/1 chamber. (b) Each GEM foil is divided into 40 and 47 high voltage sectors for short and long foils, respectively.

which is shown in Fig. 3a, using three GEM-foils arranged in the $3 / 1 / 2 / 1$ mm gap configuration with 3072 radial readout 
strips segmented over 24 readout sectors in the $(\eta, \varphi)$-plane $(\varphi$ is taken as the azimuthal angle)The GEM foils are produced by single-mask etching technique at CERN, and each foil is divided into 40 and 47 high voltage sectors that are transverse to the direction of the readout strips as shown in Fig. $3 \mathrm{~b}$ for short and long foils, respectively. The active readout area is 0.345 and $0.409 \mathrm{~m}^{2}$ for the short and long chambers, respectively. The high voltage is applied between the copper layers in order to obtain an electric field of the order of $\sim 60$ $\mathrm{kV} / \mathrm{cm}$ in the kapton and few $\mathrm{kV} / \mathrm{cm}$ in the gas gaps.

\section{GE1/1 DETECTOR DESIGN AND PERFORMANCE}

The CMS triple-GEM prototypes are produced with very innovative assembly technique based on mechanical stretching of the GEM foils. Initially, the GEM foils are tested for leakage current and the readout boards are checked with a dedicated tool capable of identifying any possible bending damage. Stainless steel nuts are embedded into the frames with the axes of their threaded holes oriented perpendicular to the inner frame and GEM foil surfaces which is shown in Fig. 4 , and this allows to assemble a full chamber in few hours time period [4].

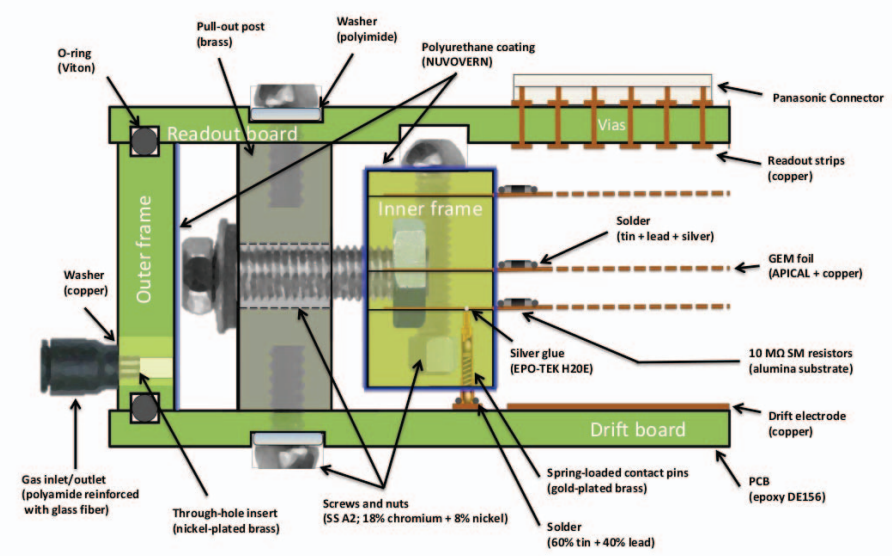

Fig. 4. Cross section through inner and outer chamber frames and GEM foils which shows how the GEM foils are mounted within the GE1/1 so that they can be mechanically tensioned against the brass pull-out posts without any deformation on the drift or readout boards.

The performance of the GE1/1 prototypes was evaluated with many test beams since 2009. The latest test beams have been performed at CERN in 2014 October (H2), 2014 November-December (H4), and 2015 October-November (H4). The chambers were operated with $\mathrm{Ar} / \mathrm{CO}_{2} / \mathrm{CF} 4$ 45:15:40 and $\mathrm{Ar} / \mathrm{CO}_{2} 70: 30$ gas mixtures with a gain around $10^{4}$, and read-out with binary output VFAT2 [5] front-end chips. An efficiency of $98 \%$ was achieved when the detector operated with the high voltage that corresponds to a gain about $10^{4}$ for both gas mixtures used during $\mathrm{H} 2$ and H4 test beam 2014 campaign. For H4 test beam in 2015 test beam, the first superchamber prototype was tested, and also one of the purposes was to test and debug some of the proposed GEM electronics readout system which is shown in Fig. 5. However, completed electronics design will start in 2016 when VFAT3 chips are available. Time response of the detector was measured as approximately $5 \mathrm{~ns}$, using both gas mixtures, $\mathrm{Ar} / \mathrm{CO}_{2} / \mathrm{CF} 4$ 45:15:40 and $\mathrm{Ar} / \mathrm{CO}_{2} 70: 30$ as shown in Fig.6 during the test beam campaign in 2014 .

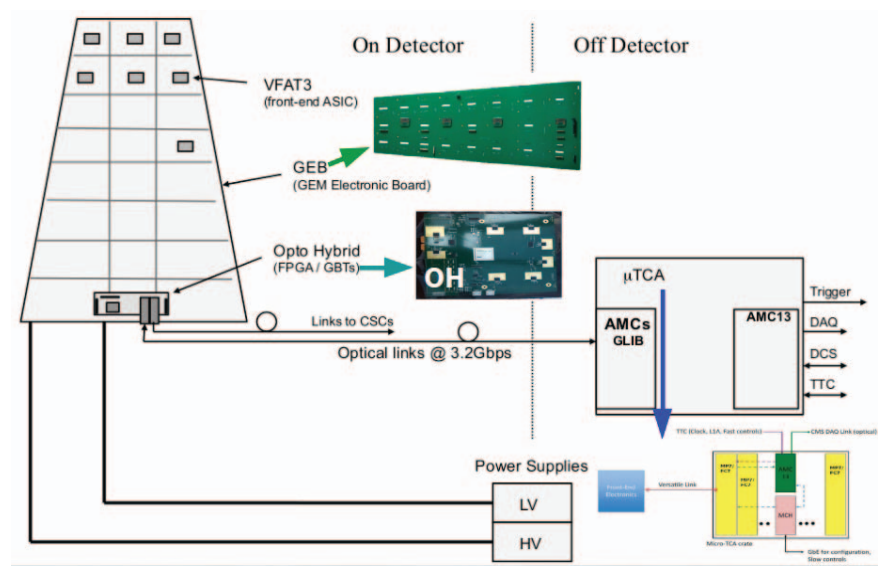

Fig. 5. New GEM electronics design with VFAT3 chip, GEB and Opto Hybrid on detector and $\mu \mathrm{TCA}$ off detector side.

Test beam measurements yield acceptable results in terms of detector performance so that the measured properties are found to be suitable for the CMS forward muon upgrade.

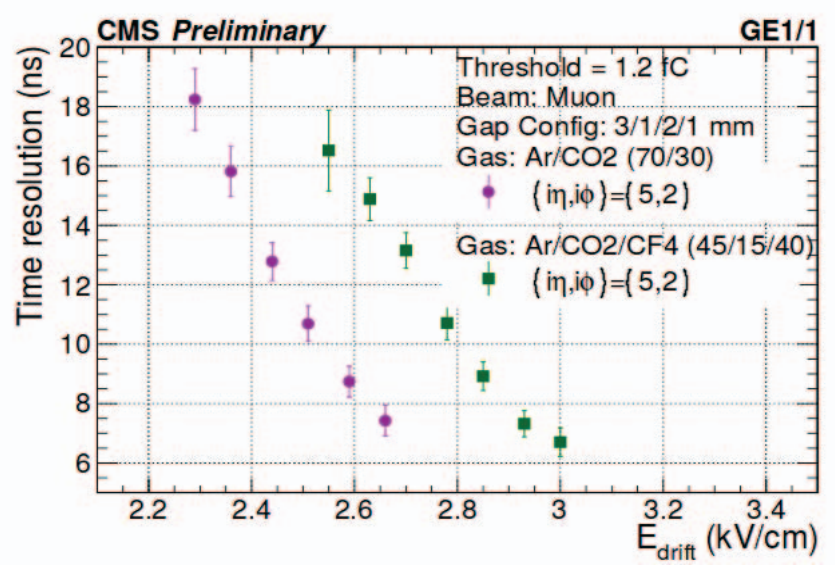

Fig. 5. Time response of the GE1/1 detector with Ar/CO2/CF4 45:15:40 and $\mathrm{Ar} / \mathrm{CO} 2$ 70:30 gas mixtures.

\section{QUALITY CONTROLS OF THE GE1/1 DETECTOR}

A common construction and quality control procedure are required to ensure the performance of each detector. The quality control steps include optical inspection, cleaning and baking of all materials and parts used to build the detector, leakage current tests of the GEM foils, high voltage tests, gas leak tests of the chambers, gain calibration to know the 
optimal operation region of the detector, gain uniformity tests, and studying the efficiency, noise and tracking performance of the detectors in a cosmic stand using scintillators. Final procedures are assembly and commissioning of the superchambers at CERN before installation in CMS, and all these steps are shown in Fig. 6. The GE1/1 chamber assembly will be done at several locations inside and outside CERN. At present, six assembly sites are being considered for GE1/1 mass production. All sites will follow the same assembly and quality control protocols, so they include same setups for assembly which needs to be installed clean room at each site, gain uniformity measurement with X-ray source, GEM foil leakage currents measurement at voltages up to $500 \mathrm{~V}$ which requires a nitrogen-flushed box, and a gas leak measurement to verify gas tightness of the chambers.
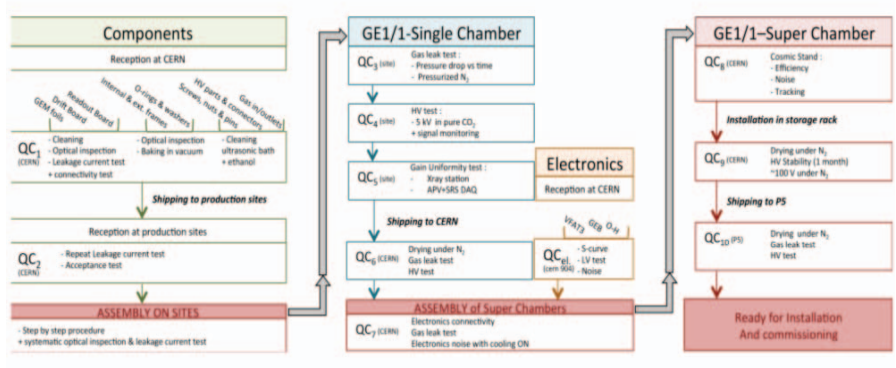

Fig. 6. Quality control steps of GE1/1 detector which include the components, tests of a single chamber and measurements on the GE1/1 superchamber.

The gain uniformity of each chamber is verified in a dedicated setup inside a copper protective box with an SRS/APV25 hybrid based data acquisition [6]. For this measurement, X-ray source is placed such that radiating the full chamber at once. The relative gain of the chamber as function the position along its surface is obtained by this measurement.

\section{SUMMARY AND CONCLUSION}

In consequence of High Luminosity phase of the LHC, the CMS Collaboration is planning several muon system upgrades in order to maintain its high level performance in terms of muon triggering and reconstruction. The installation of GE1/1 station is recently approved by the CMS Collaboration, so during LHC Long Shutdown 2, the region of $1.6<|\eta|<2.2$ of the first endcap disk will be equipped with a total of 144 new triple-GEM detectors which form 72 superchambers. Furthermore, during 2016-2017 year end technical stop of LHC, a $40^{\circ}$ wedge of GE1/1 will be installed in CMS and this operation gains experience before the full installation in LS2. The CMS triple-GEM prototypes are produced with an innovative assembly technique based on mechanical stretching of the GEM foils. The GE1/1 chamber assembly will be done at several locations inside and outside of CERN, a detailed chamber assembly and quality control work flow are being worked out. Test beam measurements yield good results in terms of the detector performance, and the results achieve the CMS requirements for the upgrade of the muon system with a new GE1/1 station. The performance of the first superchamber which was tested during the H4 2015 test beam is being studied, herewith the new electronics design including also new VFAT3 chip improvements.

\section{ACKNOWLEDGMENT}

We gratefully acknowledge support from FRS-FNRS (Belgium), FWO-Flanders (Belgium), BSF-MES (Bulgaria), BMBF (Germany), DAE (India), DST (India), INFN (Italy), NRF (Korea), QNRF (Qatar), DOE (USA).

\section{REFERENCES}

[1] F. Sauli, "GEM: A new concept for electron amplification in gas detectors", Nucl. Instrum. Meth. A 386 531, 1997.

[2] CMS collaboration, CMS The Muon Project Technical Design Report, CERN-LHCC-1008-032, CMS-TDR-3.

[3] CMS GEM Collaboration, CMS Technical Design Report for the muon endcap GEM upgrade, CMS-TDR-15-001-001.

[4] D. Abbaneo et al., Nucl. Instrum. Meth. A732, 203- 207, 2013.

[5] P. Aspell et al., "The VFAT production test platform for the TOTEM experiment", in proceedings of Workshop on Electronics for Particle Physics 2008, CERN-2008-008.

[6] S. Martoiu et al., 2011 IEEE Nucl. Sci. Symp. Conf. Rec., 2036-2038, 2011. 